\title{
Bi-Hamiltonian structure of the extended noncommutative Toda hierarchy
}

Chuanzhong Li, Tao Song

To cite this article: Chuanzhong Li, Tao Song (2016) Bi-Hamiltonian structure of the extended noncommutative Toda hierarchy, Journal of Nonlinear Mathematical Physics 23:3, 368-382, DOI: https://doi.org/10.1080/14029251.2016.1199498

To link to this article: https://doi.org/10.1080/14029251.2016.1199498

Published online: 04 January 2021 


\title{
Bi-Hamiltonian structure of the extended noncommutative Toda hierarchy
}

\author{
Chuanzhong $\mathrm{Li}$ \\ Department of Mathematics, Ningbo University \\ Ningbo, 315211, Zhejiang, P. R. China \\ Email:lichuanzhong@nbu.edu.cn. \\ Tao Song \\ Department of Mathematics, Ningbo University \\ Ningbo, 315211, Zhejiang, P. R. China
}

Received 23 January 2016

Accepted 8 May 2016

\begin{abstract}
The noncommutative Toda hierarchy is studied with the help of Moyal deformation by a reduction on the noncommutative two dimensional Toda hierarchy. Further we generalize the noncommutative Toda hierarchy to the extended noncommutative Toda hierarchy. To survey on its integrability, we construct the bi-Hamiltonian structure and noncommutative conserved densities of the extended noncommutative Toda hierarchy by means of the R-matrix formalism. This extended noncommutative Toda hierarchy can be reduced to the extended multicomponent Toda hierarchy, extended $Z_{N}$-Toda hierarchy, extended Toda hierarchy respectively by reductions on Lie algebras.
\end{abstract}

Keywords: noncommutative Toda hierarchy, extended noncommutative Toda hierarchy, bi-Hamiltonian structure.

2000 Mathematics Subject Classification: 37K05, 37K10, 37K40

\section{Introduction}

The noncommutative extension of field theories is a fruitful subject in both mathematics and physics. Noncommutative spaces contain the spatial coordinates $x^{i}$ with the noncommutativity of $\left[x^{i}, x^{j}\right]=i \theta^{i j}$, where the noncommutative parameter $\theta^{i j}$ is a anti-symmetric tensor. As in [1], the noncommutative parameter is a real constant and closely related to the existence of a background flux. The noncommutative theory gives rise to various new physical objects such as the canonical commutation relation $[q, p]=i \hbar$ in quantum mechanics.

In the effective theory of D-branes, in the presence of background magnetic fields the noncommutative gauge theories are found to be equivalent to ordinary gauge theories. In the study of Dbrane dynamics, noncommutative solitons play important roles. Such theories with noncommuting coordinates are known to emerge from limits of $M$ theory and string theory as shown in [2].

In the framework of noncommutative integrable hierarchy, infinite conserved quantities and exact soliton solutions were given for many noncommutative integrable equations in terms of Strachan's products and quasi-determinants. These noncommutative integrable equations have a close relation to an noncommutative anti-self-dual Yang-Mills theory [1]. An infinite number of conserved laws for the noncommutative Lax hierarchies were presented which include noncommutative versions of KP, KdV, Boussinesq, coupled KdV, Sawada-Kotera, modified KdV equation and so on 
in [3]. The noncommutative matrix KP hierarchy was studied in [4] from which the soliton solutions could be represented by generalized Wronskian matrices. The noncommutative KP hierarchy and the noncommutative Toda hierarchy are introduced using the Birkhoff decomposition of groups over the noncommutative algebra in $[5,6]$.

Toda equation is an important integrable equation in both physics and mathematics which has infinite conserved laws [7-9]. Toda hierarchy [10] has many kinds of reduction or extension, for example extended Toda hierarchy (ETH) [11,12], bigraded Toda hierarchy (BTH) [15]- [19] and so on. Adding additional logarithm flows to the Toda lattice hierarchy, the Toda hierarchy becomes the extended Toda hierarchy [11] which was first conjectured and then shown to govern the GromovWitten invariant of $\mathbb{C} P^{1}[13]$, by considering matrix models describing in the large $\mathrm{N}$ limit the $\mathbb{C} P^{1}$ topological sigma model [14]. That is why we think the new noncommutative logarithmic hierarchy in this paper might be useful in noncommutative Gromov-Witten invariants theory.

Noncommutative Sato theory is known to be one of the most beautiful theories of solitons and it reveals important integrable properties, such as the existence of bihamiltonian structure and hidden symmetries in noncommutative spaces. So it is reasonable and interesting to extend Sato's theory onto noncommutative spaces to clarify various integrable aspects.

Noncommutative integrable theories on the flat space are described by the replacement of ordinary products in the commutative field theory with the Moyal-products [20]. The Moyal-product can be defined for ordinary fields explicitly by

$$
\begin{aligned}
f(x) * g(x) & :=\left.\exp \left(\frac{i}{2} \theta^{\mu v} \partial_{x_{1}^{\mu}} \partial_{x_{2}^{v}}\right) f\left(x_{1}\right) g\left(x_{2}\right)\right|_{x_{1}=x_{2}=x} \\
& =f(x) g(x)+\frac{i}{2} \theta^{\mu v} \partial_{x^{\mu}} f(x) \partial_{x^{v}} g(x)+\mathscr{O}\left(\theta^{2}\right) .
\end{aligned}
$$

Here the same index means the contractive. This Moyal-product has the associativity as $f *(g * h)=$ $(f * g) * h$. In the commutative limit as $\theta^{\mu \nu} \rightarrow 0$, it will be reduced to the ordinary product. Then the product makes the ordinary coordinate noncommutative as $\left[x^{\mu}, x^{\nu}\right]_{*}:=x^{\mu} * x^{\nu}-x^{\nu} * x^{\mu}=i \theta^{\mu \nu}$. Here $x^{\mu}$ can be a spatial or time variable. A nontrivial point is that noncommutative field equations contain infinite number of derivatives. In the case of space-space noncommutativity, it is easy to find its infinite conserved laws similarly as the commutative case. For space-time noncommutativity, the integrability of the equations are not so trivial as commutative cases [3].

In this article, we will report some new noncommutative extension of Toda type integrable systems. Further we give their bi-Hamiltonian structures in the framework of extended noncommutative Toda hierarchy.

\section{Noncommutative 2D Toda hierarchy}

In this section, we will firstly recall the theory of Lie algebra factorization in noncommutative version which was discussed in detail in [21]. Let $\left(t_{1}, t_{2}, \cdots\right)$ be coordinates of noncommutative plane $\mathbb{R}^{2 \infty}$ which satisfy $\left[t_{n}, t_{m}\right]_{*}=i \theta_{n m}$, and $\mathscr{A}_{\theta}$ a set of functions on it. The deformation parameters $\theta_{n m} \in \mathbb{R}$ are assumed to be non-zero constants and a matrix $\left(\theta_{n m}\right)$ is invertible.

Here we introduce the noncommutative Toda hierarchy as [6]. Firstly we need one more series of coordinates $\left(\bar{t}_{1}, \bar{t}_{2}, \cdots\right)$ which satisfy $\left[\bar{t}_{n}, \bar{t}_{m}\right]_{*}=i \bar{\theta}_{n m},\left[\bar{t}_{n}, t_{m}\right]_{*}=0$ and $\mathscr{A}_{\theta, \bar{\theta}}$ denotes a set of functions on it. Let $S(\bar{S})$ be an difference operator whose components take values in $\mathscr{A}_{\theta, \bar{\theta}}$. The Moyal-product 
for the noncommutative Toda hierarchy can be defined for ordinary fields explicitly by

$$
f(t, \bar{t}) * g(t, \bar{t}):=\left.\exp \left(\frac{i}{2}\left(\theta_{\mu \nu} \partial_{x_{\mu}} \partial_{y_{v}}+\bar{\theta}_{\mu v} \partial_{\bar{x}_{\mu}} \partial_{\bar{y}_{v}}\right)\right) f(x, \bar{x}) g(y, \bar{y})\right|_{x=y=t, \bar{x}=\bar{y}=\bar{t}} .
$$

In this section, we will denote $G$ as a group which contains invertible elements of complex noncommutative functions under the moyal product and denote its Lie algebra $\mathfrak{g}$ as the associative algebra of complex noncommutative functions. Now we will consider the linear space of functions $g: \mathbb{R} \rightarrow \mathbb{C}$ with the shift operator $\Lambda$ acting on any function $g(x)$ as $(\Lambda g)(x):=g(x+\varepsilon)$.

This Lie algebra as a linear space has the following important splitting

$$
\mathfrak{g}=\mathfrak{g}_{+} \oplus \mathfrak{g}_{-},
$$

where

$$
\mathfrak{g}_{+}=\left\{\sum_{j \geq 0} X_{j}(x) \Lambda^{j}, \quad X_{j}(x) \in \mathfrak{g}\right\}, \quad \mathfrak{g}_{-}=\left\{\sum_{j<0} X_{j}(x) \Lambda^{j}, \quad X_{j}(x) \in \mathfrak{g}\right\} .
$$

The splitting (2.2) leads us to consider the following factorization of $g \in G$

$$
g=g_{-}^{-1} * g_{+}, \quad g_{ \pm} \in G_{ \pm}
$$

where $G_{ \pm}$have $\mathfrak{g}_{ \pm}$as their Lie algebras. Here $G_{+}$is the set of invertible linear operators of the form $\sum_{j \geq 0} g_{j}(x) \Lambda^{j}$; while $G_{-}$is the set of invertible linear operators in the form of $1+\sum_{j<0} g_{j}(x) \Lambda^{j}$.

The Sato-Wilson equations of the noncommutative Toda hierarchy can be defined as

$$
\begin{aligned}
& \frac{\partial S}{\partial t_{n}}=-\left(S * \Lambda^{n} * S^{-1}\right)_{-} * S, \frac{\partial S}{\partial \bar{t}_{n}}=-\left(\bar{S} * \Lambda^{-n} * \bar{S}^{-1}\right)_{-} * S, \\
& \frac{\partial \bar{S}}{\partial t_{n}}=\left(S * \Lambda^{n} * S^{-1}\right)_{+} * \bar{S}, \frac{\partial \bar{S}}{\partial \bar{t}_{n}}=\left(\bar{S} * \Lambda^{-n} * \bar{S}^{-1}\right)_{+} * \bar{S},
\end{aligned}
$$

for $n \geq 1$. For a noncommutative function $P,(P)_{+}$denotes the nonnegative powers' projection


terms of the shift operator $\Lambda$. Note that these Sato equations are equivalent to the Lax equations and the Zakharov-Shabat equations. If one define $b(x), c(x) \in \mathscr{A}_{\theta, \bar{\theta}}$ as $B:=\left(S \Lambda S^{-1}\right)_{+}=\Lambda+b(x)$ and $C:=\left(S \Lambda^{-1} S^{-1}\right)_{-}=c(x) \Lambda^{-1}$. The Zakharov-Shabat equation $\frac{\partial B}{\partial \bar{t}_{1}}-\frac{\partial C}{\partial t_{1}}+[B, C]_{*}=0$ gives the noncommutative Toda equations $[6,22,23]$

$$
\frac{\partial b(x)}{\partial \bar{t}_{1}}=c(x)-c(x+\varepsilon), \quad \frac{\partial c(x)}{\partial t_{1}}=b(x) * c(x)-c(x) * b(x-\varepsilon) .
$$

For any function $f(x) \in \mathscr{A}(\boldsymbol{\theta}, \bar{\theta})$, its exponential is defined as

$$
e_{*}^{f(x)}=1+\sum_{i=1}^{\infty} \frac{1}{n !} \underbrace{f(x) * f(x) * \cdots * f(x)}_{n \text { times }} .
$$

Let $w_{n}(x), \bar{w}_{n}(x), w_{n}^{*}(x), \bar{w}_{n}^{*}(x) \in \mathscr{A}_{\theta, \bar{\theta}}$ be operators which are coefficients of $S, \bar{S}, S^{-1}, \bar{S}^{-1}$

$$
S=\sum_{n \geq 0} w_{-n}(x) \Lambda^{-n}, \quad S^{-1}=\sum_{n \geq 0} \Lambda^{-n} w_{-n}^{*}(x) .
$$




$$
\bar{S}=\sum_{n \geq 0} \bar{w}_{n}(x) \Lambda^{n}, \quad \bar{S}^{-1}=\sum_{n \geq 0} \Lambda^{n} \bar{w}_{n}^{*}(x) .
$$

One need to note that the inverses $S^{-1}, \bar{S}^{-1}$ are under the Moyal deformation, i.e. $S * S^{-1}=$ $\bar{S} * \bar{S}^{-1}=1$. We introduce the operator valued Baker-Akhiezer functions as [6]

$$
\begin{aligned}
& w(x, \lambda)=\left(\sum_{n \geq 0} w_{-n}(x) \lambda^{-n+s}\right) e_{*}^{\hat{\xi}_{-}}, \quad \bar{w}(x, \lambda)=\left(\sum_{n \geq 0} \bar{w}_{n} \lambda^{n+s}\right) e_{*}^{\hat{\xi}_{+}}, \\
& w^{*}(x, \lambda)=e_{*}^{-\hat{\xi}_{-}}\left(\sum_{n \geq 0} w_{-n}^{*}(x) \lambda^{-n-s}\right), \quad \bar{w}^{*}(x, \lambda)=e_{*}^{-\hat{\xi}_{+}}\left(\sum_{n \geq 0} \bar{w}_{n}^{*} \lambda^{n-s}\right),
\end{aligned}
$$

where $\hat{\xi}_{-}:=\sum_{n \geq 1} t_{n} \lambda^{n}$ and $\hat{\xi}_{+}:=\sum_{n \geq 1} \bar{t}_{n} \lambda^{-n}$. In this commutative case, the BA functions satisfy the following bilinear equation as [6]

$$
\oint \frac{d \lambda}{2 \pi i} w\left(x^{\prime}, \lambda\right) * w^{*}(x, \lambda)=\oint \frac{d \lambda}{2 \pi i} \bar{w}\left(x^{\prime}, \lambda\right) * \bar{w}^{*}(x, \lambda) .
$$

The BA functions $\bar{w}$ and $w$ satisfy the following linear differential equations of $t_{n}$ and $\bar{t}_{n}$,

$$
\begin{array}{ll}
\frac{\partial w}{\partial t_{n}}=\left(S * \Lambda^{n} * S^{-1}\right)_{+} * w, & \frac{\partial w}{\partial \bar{t}_{n}}=\left(\bar{S} * \Lambda^{-n} * \bar{S}^{-1}\right)_{-} * w, \\
\frac{\partial \bar{w}}{\partial t_{n}}=\left(S * \Lambda^{n} * S^{-1}\right)_{+} * \bar{w}, & \frac{\partial \bar{w}}{\partial \bar{t}_{n}}=\left(\bar{S} * \Lambda^{-n} * \bar{S}^{-1}\right)_{-} * \bar{w} .
\end{array}
$$

When $\theta_{m n}=0$, the noncommutative two dimensional Toda hierarchy will be exactly the ordinary well-known two dimensional Toda hierarchy in [10].

Basing on the above defined noncommutative two dimensional Toda hierarchy, a further reduction and extension will lead to the extended noncommutative Toda hierarchy which will be talked about in the next section.

\section{Extended noncommutative Toda hierarchy}

In this section, we also use two series of coordinates $\left(t_{0}, t_{1}, t_{2}, \cdots\right),\left(s_{0}, s_{1}, s_{2}, \cdots\right)$ which satisfy $\left[t_{n}, t_{m}\right]_{*}=i \theta_{n m},\left[s_{n}, s_{m}\right]_{*}=i \Omega_{n m},\left[s_{n}, t_{m}\right]_{*}=0$ and $\mathscr{A}_{\theta, \Omega}$ denotes a set of functions on it. Here $\left(s_{0}, s_{1}, s_{2}, \cdots\right)$ will denote another extended logarithmic time variables with the first variable $s_{0}$ equivalent to the spatial variable $x$. The Moyal-product for the extended noncommutative Toda hierarchy can be defined for ordinary fields explicitly by

$$
f(t, s) * g(t, s):=\left.\exp \left(\frac{i}{2}\left(\theta_{\mu \nu} \partial_{x_{\mu}} \partial_{y_{v}}+\bar{\Omega}_{\mu v} \partial_{\bar{x}_{\mu}} \partial_{\bar{y}_{v}}\right)\right) f(x, \bar{x}) g(y, \bar{y})\right|_{x=y=t, \bar{x}=\bar{y}=s},
$$

where the spatial variable $x$ hides inside the variable sequence $s$ as $s_{0}$. Let $S(\bar{S})$ be an difference operator whose components take values in $\mathscr{A}_{\theta, \Omega}$. To define the extended Toda flows in the noncommutative space, we define the following constrained Lax operator

$$
\mathscr{L}=S * \Lambda * S^{-1}=\bar{S} * \Lambda^{-1} * \bar{S}^{-1}=\Lambda+u+v \Lambda^{-1} .
$$


To define the extended flows, we need to define the following noncommutative logarithmic operators

$$
\begin{aligned}
& \log _{+} \mathscr{L}=S * \varepsilon \partial * S^{-1}, \\
& \log _{-} \mathscr{L}=-\bar{S} * \varepsilon \partial * \bar{S}^{-1},
\end{aligned}
$$

where $\partial$ is the derivative about spatial variable $x$.

Combining these above logarithm operators together can derive the following important noncommutative logarithm

$$
\log \mathscr{L}:=\frac{1}{2}\left(\log _{+} \mathscr{L}+\log _{-} \mathscr{L}\right)=\frac{1}{2}\left(S * \varepsilon \partial * S^{-1}-\bar{S} * \varepsilon \partial * \bar{S}^{-1}\right):=\sum_{i=-\infty}^{+\infty} W_{i} \Lambda^{i} \in G,
$$

which will generate a series of flow equations which contain the spatial flow in later defined Lax equations. Let us first introduce some convenient noncommutative notations.

Definition 3.1. The noncommutative operators $B_{j}, D_{j}$ are defined as follows

$$
B_{j}:=\frac{\mathscr{L}^{j+1}}{(j+1) !}, \quad D_{j}:=\frac{2 \mathscr{L}^{j}}{j !} *\left(\log \mathscr{L}-c_{j}\right), \quad c_{j}=\sum_{i=1}^{j} \frac{1}{i}, j \geq 0 .
$$

Now we give the definition of the extended noncommutative Toda hierarchy(ENTH).

Definition 3.2. The extended noncommutative Toda hierarchy is a hierarchy in which the noncommutative dressing operators $S, \bar{S}$ satisfy following noncommutative Sato equations

$$
\begin{array}{ll}
\varepsilon \partial_{t_{j}} S=-\left(B_{j}\right)_{-} * S, & \varepsilon \partial_{t_{j}} \bar{S}=\left(B_{j}\right)_{+} * \bar{S}, \\
\varepsilon \partial_{s_{j}} S=-\left(D_{j}\right)_{-} * S, & \varepsilon \partial_{s_{j}} \bar{S}=\left(D_{j}\right)_{+} * \bar{S} .
\end{array}
$$

Similarly as the proof in [3], we can prove the above flows of the ENTH can commute with each other. From the previous definition we derive the following Lax equations for the Lax operators.

Proposition 3.1. The Lax equations of the ENTH are as follows

$$
\begin{gathered}
\varepsilon \partial_{t_{j}} \mathscr{L}=\left[\left(B_{j}\right)_{+}, \mathscr{L}\right]_{*}, \quad \varepsilon \partial_{s_{j}} \mathscr{L}=\left[\left(D_{j}\right)_{+}, \mathscr{L}\right]_{*}, \varepsilon \partial_{t_{j}} \log \mathscr{L}=\left[\left(B_{j}\right)_{+}, \log \mathscr{L}\right]_{*}, \\
\varepsilon(\log \mathscr{L})_{s_{j}}=\left[-\left(D_{j}\right)_{-}, \frac{1}{2} \log _{+} \mathscr{L}\right]_{*}+\left[\left(D_{j}\right)_{+}, \frac{1}{2} \log _{-} \mathscr{L}\right]_{*} .
\end{gathered}
$$

The extended flow can be equivalently written as

$$
\tilde{D}_{j}=\frac{\mathscr{L}^{j}}{j !} \varepsilon \partial+\left[\frac{\mathscr{L}^{j}}{j !} *\left(2 \sum_{i=-\infty}^{-1} W_{i} \Lambda^{i}-c_{j}\right)\right]_{+}-\left[\frac{\mathscr{L}^{j}}{j !} *\left(2 \sum_{i=0}^{\infty} W_{i} \Lambda^{i}-c_{j}\right)\right]_{-} .
$$

To see the extended noncommutative Toda equations clearly, we will give several simple equations as following 


$$
\begin{aligned}
& \varepsilon \partial_{s_{0}} \mathscr{L}=\left[\left(D_{0}\right)_{+}, \mathscr{L}\right]_{*}=\varepsilon \partial_{x} \mathscr{L}, \\
& \varepsilon \partial_{s_{1}} \mathscr{L}=\left[\left(D_{1}\right)_{+}, \mathscr{L}\right]_{*}=\left[\frac{\Lambda}{1-\Lambda} u_{x}-2 v * W_{0} \Lambda^{-1}+2 \mathscr{L} \varepsilon \partial-2 \mathscr{L}_{+}, \mathscr{L}\right]_{*} .
\end{aligned}
$$

One can find that the first variable $s_{0}$ is equivalent to the spatial variable $x$, therefore we can treat them being the same thing. In other words, every function related to the ENTH should depend on $s_{0}+x$ as a linear combination. By considering the relation among coefficients as

$$
W_{0}=-\tilde{w}_{0 x} * \tilde{w}_{0}^{-1}, \quad v * \tilde{w}_{0}(x-\varepsilon)=\tilde{w}_{0}, \quad v * W_{0}=-v * \tilde{w}_{0 x} * \tilde{w}_{0}^{-1},
$$

we can get the noncommutative equations as

$$
\begin{aligned}
& \varepsilon \partial_{s_{1}} u=\left[\frac{\Lambda}{1-\Lambda} u_{x}, u\right]_{*}-2 \partial_{t_{1}} u \\
& \varepsilon \partial_{s_{1}} v=\left(\frac{\Lambda}{1-\Lambda} u_{x}\right) * v-v *\left(\frac{1}{1-\Lambda} u_{x}\right)-2 \partial_{t_{1}} v
\end{aligned}
$$

Remark 3.1. The spatial variable $x$ which is equivalent to the $s_{0}$ can commute with the $t_{i}$ flow of the ENTH. Whether $x$, i.e. $s_{0}$ can commute with other extended logarithmic variables $s_{v}$ depends on whether $\Omega_{0 v}$ is zero for every $v$.

To prove the integrability, we will construct the bi-Hamiltonian structure of the extended noncommutative Toda hierarchy in the next section.

\section{Bi-Hamiltonian structure of the ENTH}

As we know, bi-Hamiltonian structures are important properties of integrable systems [5]. For a noncommutative sequence $A=\Lambda+a+b \Lambda^{-1}$, the vector field $\partial_{A}$ over ENTH is defined by

$$
\partial_{A}=\sum_{k \in \mathbb{Z}} a(x+k \varepsilon) * \frac{\partial}{\partial u(x+k \varepsilon)}+b(x+k \varepsilon) * \frac{\partial}{\partial v(x+k \varepsilon)} .
$$

For two functionals $\bar{f}=\int f(u, v) d^{D} x, \bar{g}=\int g(u, v) d^{D} x$, we have

$$
\partial_{A} \bar{f}=\int \sum_{k \in \mathbb{Z}} a(x+k \varepsilon) * \frac{\partial f}{\partial u(x+k \varepsilon)}+b(x+k \varepsilon) * \frac{\partial f}{\partial v(x+k \varepsilon)} d^{D} x .
$$

Remark 4.1. The integral directions $d^{D} x$ are chosen in $x$ and all directions which are noncommutative with $x=s_{0}$. That means that the directions $d^{D} x$ in the integration depend on the choice of time/spatial variables and the matrix $\theta$ and $\Omega$. In physics, one always choose some variables as spatial variables and someone as the time variable. If you only study on the $t_{i}$ flows as time flows, i.e. the case of space-space noncommutativity (if you only consider $x=s_{0}$ as a sole spatial variable, then it is commutative), the integration will take only in the spatial direction $d x$ as the commutative case. If you only study on the $s_{i}$ flows as time flows, i.e. the case of space-time noncommutativity $\left(\Omega_{0 j} \neq 0\right.$ for some index $j$ ), the integration will take only in the directions $x$ and $s_{j}$. 
To show why we make the above remark, it is convenient to define the following Strachan product as [3],

$$
f(x, t, s) \diamond g(x, t, s):=f(x, t, s) \sum_{s=0}^{\infty} \frac{(-1)^{s}}{(2 s+1) !}\left[\left(\overleftarrow{\partial_{t_{i}}} \theta^{i j} \overrightarrow{\partial_{t_{j}}}\right)^{2 s}+\left(\overleftarrow{\partial_{s_{i}}} \Omega^{i j} \overrightarrow{\partial_{s_{j}}}\right)^{2 s}\right] g(x, t, s)
$$

where $\overleftarrow{\partial_{i}}$ means taking derivative backward and $\overrightarrow{\partial_{j}}$ means taking derivative forward. Then a commutator can be straightforwardly calculated as follows

$$
[f(x, t, s), g(x, t, s)]_{*}=-\theta^{i j} \partial_{t_{i}}\left(f(x, t, s) \diamond \partial_{t_{j}} g(x, t, s)\right)-\Omega^{i j} \partial_{s_{i}}\left(f(x, t, s) \diamond \partial_{s_{j}} g(x, t, s)\right) .
$$

For any difference operator $A=\sum_{k} A_{k} \Lambda^{k}$, define residue $\operatorname{Res} A=A_{0}$.

Definition 4.1. Let $M=M(u, v)$ be a suitable manifold possessing a manifold variable $(u, v)$. For any $\bar{f}=\int f(u, v) d^{D} x$ with $f(u, v) \in C^{\infty}(M)$, its variational derivatives $d \bar{f}:=\frac{\delta \bar{f}}{\delta u}+\Lambda \circ \frac{\delta \bar{f}}{\delta v}$ are defined by

$$
\partial_{A} \bar{f}=\langle A, d \bar{f}\rangle:=\operatorname{Res} A * d \bar{f}
$$

where

$$
\langle A, d \bar{f}\rangle=\int a(x) * \frac{\delta \bar{f}}{\delta u(x)}+b(x) * \frac{\delta \bar{f}}{\delta v(x)} d^{D} x .
$$

One can easily derive the formula of the variational derivatives $\left(\frac{\delta \bar{f}}{\delta w}, w=u\right.$ or $\left.v\right)$ as

$$
\frac{\delta \bar{f}}{\delta w(x)}=\sum_{k \in \mathbb{Z}} \Lambda^{-k}\left(\frac{\partial f}{\partial w(x+k \varepsilon)}\right), w=u \text { or } v .
$$

Then we can define the Hamiltonian bracket of the ENTH as

$$
\{\bar{f}, \bar{g}\}=\int \sum_{w, w^{\prime}} \frac{\delta \bar{f}}{\delta w} *\left\{w, w^{\prime}\right\} * \frac{\delta \bar{g}}{\delta w^{\prime}} d^{D} x=<d \bar{f}, J(d \bar{g})>, w, w^{\prime}=u \text { or } v,
$$

where $d \bar{f}, d \bar{g}$ are two difference operators that represent the differentials of the functions $\bar{f}, \bar{g}$ and the Hamiltonian operator $J$ maps 1-forms to vectors.

The bi-Hamiltonian structure for the ENTH can be given by the following two compatible Poisson structures which is a noncommutative generalization of the extended Toda hierarchy in [11]

Proposition 4.1. The following formulas

$$
\begin{aligned}
J_{1}(X)= & \frac{1}{\varepsilon}\left[X_{+}, \mathscr{L}\right]_{* \leq 0}-\frac{1}{\varepsilon}\left[X_{-}, \mathscr{L}\right]_{*>0} \\
J_{2}(X)= & \frac{1}{2 \varepsilon}\left[\mathscr{L},(\mathscr{L} * X+X * \mathscr{L})_{-}\right]_{*}-\frac{1}{2 \varepsilon} \mathscr{L} *[\mathscr{L}, X]_{* \leq 0}-\frac{1}{2 \varepsilon}[\mathscr{L}, X]_{* \leq 0} * \mathscr{L} \\
& +\frac{1}{2 \varepsilon}\left[\mathscr{L},\left((\Lambda+1)(\Lambda-1)^{-1} \operatorname{Res}[L, X]_{*}\right)\right]_{*}
\end{aligned}
$$


define two compatible Hamiltonian operators. That means that the brackets $\{,\}_{1}$ and $\{,\}_{2}$ defined by

$$
\{f, g\}_{i}=<d f, J_{i} d g>
$$

are two compatible Poisson brackets as following

$$
\begin{aligned}
\{u(x), u(y)\}_{1}= & \frac{1}{\varepsilon}\left[R_{u}-L_{u}\right] \delta(x-y), \\
\{u(x), v(y)\}_{1}= & \frac{1}{\varepsilon}\left[\Lambda R_{v}-L_{v}\right] \delta(x-y), \\
\{v(x), v(y)\}_{1}= & 0 \\
\{u(x), u(y)\}_{2}= & \frac{1}{\varepsilon}\left[\Lambda R_{v}-v * \Lambda^{-1}+R_{u} *\left(\frac{\Lambda}{\Lambda-1} R_{u}\right)\right. \\
& \left.-\frac{\Lambda}{\Lambda-1} * u * R_{u}-u *(\Lambda-1)^{-1} R_{u}+u *(\Lambda-1)^{-1} u\right] \delta(x-y), \\
\{u(x), v(y)\}_{2}= & \frac{1}{\varepsilon}\left[R_{u} * \frac{\Lambda}{\Lambda-1} * R_{v(x+\varepsilon)}-R_{u} \frac{\Lambda}{\Lambda-1} * v * \Lambda^{-1}\right. \\
& \left.-u *(\Lambda-1)^{-1} * R_{v(x+\varepsilon)}+u *(\Lambda-1)^{-1} * v \Lambda^{-1}\right] \delta(x-y), \\
\{v(x), v(y)\}_{2}= & \frac{1}{\varepsilon}\left[R_{v} * \Lambda^{2}(\Lambda-1)^{-1} * R_{v}-R_{v} * \frac{\Lambda}{\Lambda-1} * v\right. \\
& \left.-v *(\Lambda-1)^{-1} * R_{v}+v * \Lambda^{-1}(\Lambda-1)^{-1} * v\right] \delta(x-y) .
\end{aligned}
$$

Here $L_{u}, L_{v}$ means the multiplication by $u, v$ respectively from the left side and $R_{u}, R_{v}$ means the multiplication by $u, v$ respectively from the right side.

Proof. The fact that $\{,\}_{1}$ and $\{,\}_{2}$ are two compatible Poisson brackets follows from the $R$-matrix theory in [27] with the following basic steps. We consider the associative algebra $\mathfrak{g}$ of difference operators with arbitrary upper-bounded order

$$
\mathfrak{g}=\left\{X=\sum_{k<\infty} u_{k} \Lambda^{k}\right\}
$$

and identify with its dual $\mathfrak{g}^{*}$ using the non-degenerate invariant inner product (4.5). The map $R: \mathfrak{g} \rightarrow$ $\mathfrak{g}$ by $R(X)=X_{+}-X_{-}$and its skew-symmetric part $A=\frac{1}{2}\left(R-R^{*}\right)$ satisfy the modified Yang-Baxter equation similarly as the proof in [15]

$$
[R(X), R(Y)]_{*}-R\left([R(X), Y]_{*}+[X, R(Y)]_{*}\right)=-[X, Y]_{*} \quad \text { for any } X, Y \in \mathfrak{g} .
$$

Then we can derive

$$
\begin{aligned}
J_{1} d u(y) & =\frac{1}{\varepsilon}[\delta(x-y), \mathscr{L}(x)]_{* \leq 0} \\
& =\frac{1}{\varepsilon} \delta(x-y) * u(x)-\frac{1}{\varepsilon} u(x) * \delta(x-y)+\frac{1}{\varepsilon} \delta(x-y) * v(x) \Lambda^{-1}-\frac{1}{\varepsilon} v(x) * \delta(x-\varepsilon-y) \Lambda^{-1}, \\
J_{1} d v(y) & =\frac{1}{\varepsilon}[\delta(x+\varepsilon-y) \Lambda, \mathscr{L}(x)]_{* \leq 0}=\frac{1}{\varepsilon} \delta(x+\varepsilon-y) * v(x+\varepsilon)-\frac{1}{\varepsilon} v(x) * \delta(x-y),
\end{aligned}
$$


which further leads to

$$
\begin{aligned}
\{u(x), u(y)\}_{1} & =\left\langle d u(x), J_{1} d u(y)\right\rangle \\
& =\left\langle 1, J_{1} d u(y)\right\rangle=\frac{1}{\varepsilon} \delta(x-y) * u(x)-\frac{1}{\varepsilon} u(x) * \delta(x-y), \\
\{u(x), v(y)\}_{1} & =\left\langle d u(x), J_{1} d v(y)\right\rangle=\frac{1}{\varepsilon}\left[\Lambda R_{v}-L_{v}\right] \delta(x-y), \\
\{v(x), v(y)\}_{1} & =\left\langle d v(x), J_{1} d v(y)\right\rangle \\
& =\left\langle\Lambda, J_{1} d v(y)\right\rangle=0 .
\end{aligned}
$$

Because of the non-commutativity, one can see the difference between the ENTH and the extended Toda hierarchy [11]. For the extended Toda hierarchy, the bracket $\{u(x), u(y)\}_{1}$ becomes trivial (see in [11]).

Similarly, we can derive the equations (4.15)-(4.17) by a tedious calculation from (4.10).

Finally the compatibility of $\{,\}_{1}$ and $\{,\}_{2}$ after a Dirac reduction follows from the simple observation that

$$
\{,\}_{2} \rightarrow\{,\}_{2}+\lambda\{,\}_{1}
$$

under $u \rightarrow u+\lambda$.

As another important part of the Sato theory, the Bihamiltonian equation of the ENTH will be constructed in the next section similar as [11].

Theorem 4.1. The flows of the ENTH are Hamiltonian systems of the form

$$
\frac{\partial u}{\partial t_{k, j}}=\left\{u, H_{k, j}\right\}_{1}, \frac{\partial v}{\partial t_{k, j}}=\left\{v, H_{k, j}\right\}_{1}, \quad k=0,1 ; j \geq 0,
$$

with $t_{0, j}=t_{j}, t_{1, j}=s_{j}$. They satisfy the following bi-Hamiltonian recursion relation

$$
\left\{\cdot, H_{1, n-1}\right\}_{2}=n\left\{\cdot, H_{1, n}\right\}_{1}+2\left\{\cdot, H_{0, n-1}\right\}_{1},\left\{\cdot, H_{0, n-1}\right\}_{2}=(n+1)\left\{\cdot, H_{0, n}\right\}_{1} .
$$

Here the Hamiltonians have the form

$$
H_{k, j}=\int h_{k, j}\left(u, v ; u_{x}, v_{x} ; \ldots ; \varepsilon\right) d^{D} x, \quad k=0,1 ; j \geq 0
$$

with

$$
h_{0, j}=\frac{1}{(j+1) !} \operatorname{Res} \mathscr{L}^{j+1}, h_{1, j}=\frac{2}{j !} \operatorname{Res}\left[\mathscr{L}^{j}\left(\log \mathscr{L}-c_{j}\right)\right]
$$

Proof. Here we will firstly prove that the flows $\frac{\partial}{\partial t_{1, n}}$ are Hamiltonian systems with respect to the first Poisson bracket. Similar as in [11] and by considering eq.(4.4), we can prove the following identity 


$$
\operatorname{Res}\left(\mathscr{L}^{n} d \log _{+} \mathscr{L}\right) \sim \operatorname{Res}\left(\mathscr{L}^{n-1} d \mathscr{L}\right)
$$

and

$$
\operatorname{Res}\left(\mathscr{L}^{n} d \log _{-} \mathscr{L}\right) \sim \operatorname{Res}\left(\mathscr{L}^{\mathrm{n}-1} \mathrm{~d} \mathscr{L}\right)
$$

Here the equivalent relation $\sim$ is up to derivatives for all directions corresponding to non-vanishing $\theta_{i j}$.

Combining (4.31) with (4.32) together can lead to

$$
\operatorname{Res}\left(\mathscr{L}^{n} d \log \mathscr{L}\right) \sim \operatorname{Res}\left(\mathscr{L}^{\mathrm{n}-1} \mathrm{~d} \mathscr{L}\right)
$$

Then from

$$
\frac{\partial \mathscr{L}}{\partial t_{k, n}}=\left[\left(B_{k, n}\right)_{+}, \mathscr{L}\right]_{*}=\left[-\left(B_{k, n}\right)_{-}, \mathscr{L}\right]_{*}, B_{0, n}=B_{n}, B_{1, n}=D_{n},
$$

and supposing

$$
B_{1, n}=\sum_{k} a_{1, n ; k} \Lambda^{k}
$$

we can derive the equation

$$
\begin{aligned}
& \varepsilon \frac{\partial u}{\partial t_{1, n}}=a_{1, n ; 1}(x) * v(x+\varepsilon)-v(x) * a_{1, n ; 1}(x-\varepsilon)+\left[a_{1, n ; 0}(x), u(x)\right]_{*}, \\
& \varepsilon \frac{\partial v}{\partial t_{1, n}}=a_{1, n ; 0}(x) * v(x)-v(x) * a_{1, n ; 0}(x-\varepsilon) .
\end{aligned}
$$

The equivalence relation (4.33) now follows from the above two equations. By using (4.31) we obtain

$$
\begin{aligned}
& d h_{1, n}=\frac{2}{n !} d \operatorname{Res}\left[\mathscr{L}^{n}\left(\log \mathscr{L}-c_{n}\right)\right] \\
& \sim \frac{2}{(n-1) !} \operatorname{Res}\left[\mathscr{L}^{n-1}\left(\log \mathscr{L}-c_{n}\right) d \mathscr{L}\right]+\frac{2}{n !} \operatorname{Res}\left[\mathscr{L}^{n-1} d \mathscr{L}\right] \\
& =\frac{2}{(n-1) !} \operatorname{Res}\left[\mathscr{L}^{n-1}\left(\log \mathscr{L}-c_{n-1}\right) d \mathscr{L}\right] \\
& =a_{1, n ; 0}(x) * d u+a_{1, n ; 1}(x-\varepsilon) * d v .
\end{aligned}
$$

It yields the following identities

$$
\frac{\delta H_{1, n}}{\delta u}=a_{1, n ; 0}(x), \quad \frac{\delta H_{1, n}}{\delta v}=a_{1, n ; 1}(x-\varepsilon) .
$$

This agrees with Lax equation 


$$
\begin{aligned}
\frac{\partial u}{\partial t_{1, n}}= & \left\{u, H_{1, n}\right\}_{1}=\frac{1}{\varepsilon}\left[R_{u}-L_{u}\right] \frac{\delta H_{1, n}}{\delta u}+\frac{1}{\varepsilon}\left[\Lambda R_{v}-L_{v}\right] \frac{\delta H_{1, n}}{\delta v} \\
& =\frac{1}{\varepsilon}\left[R_{u}-L_{u}\right] a_{1, n ; 0}(x)+\frac{1}{\varepsilon}\left[\Lambda R_{v}-L_{v}\right] a_{1, n ; 1}(x-\varepsilon) \\
& =a_{1, n ; 1}(x) * v(x+\varepsilon)-v(x) * a_{1, n ; 1}(x-\varepsilon)+\left[a_{1, n ; 0}(x), u(x)\right]_{*}, \\
\frac{\partial v}{\partial t_{1, n}}= & \left\{v, H_{1, n}\right\}_{1}=\frac{1}{\varepsilon}\left[R_{v}-L_{v} \Lambda^{-1}\right] \frac{\delta H_{1, n}}{\delta u}=\frac{1}{\varepsilon}\left[a_{1, n ; 0}(x) * v(x)-v(x) * a_{1, n ; 0}(x-\varepsilon)\right]
\end{aligned}
$$

From the above identities we see that the flows $\frac{\partial}{\partial t_{1, n}}$ are Hamiltonian systems of the first biHamiltonian structure. For the case of logarithmic flows the recursion relation follows from the following trivial identities

$$
\begin{aligned}
& n \frac{2}{n !} \mathscr{L}^{n}\left(\log \mathscr{L}-c_{n}\right)=\mathscr{L} \frac{2}{(n-1) !} \mathscr{L}^{n-1}\left(\log \mathscr{L}-c_{n-1}\right)-2 \frac{1}{n !} \mathscr{L}^{n} \\
& =\frac{2}{(n-1) !} \mathscr{L}^{n-1}\left(\log \mathscr{L}-c_{n-1}\right) \mathscr{L}-2 \frac{1}{n !} \mathscr{L}^{n}
\end{aligned}
$$

Then we get,

$$
\begin{aligned}
& n a_{1, n+1 ; 1}(x)=a_{1, n ; 0}(x+\varepsilon)+u * a_{1, n ; 1}(x)+v * a_{1, n ; 2}(x-\varepsilon)-2 a_{0, n ; 1}(x) \\
& =a_{1, n ; 0}(x)+a_{1, n ; 1}(x) * u(x+\varepsilon)+a_{1, n ; 2}(x) * v(x+2 \varepsilon)-2 a_{0, n ; 1}(x) .
\end{aligned}
$$

Then we also get

$$
\begin{aligned}
& n a_{1, n ; 0}(x)=a_{1, n-1 ;-1}(x+\varepsilon)+u(x) * a_{1, n-1 ; 0}(x)+v(x) * a_{1, n-1 ; 1}(x-\varepsilon)-\frac{2}{n !} a_{0, n ; 0}(x) \\
& =a_{1, n-1 ;-1}(x)+a_{1, n-1 ; 0}(x) * u(x)+a_{1, n-1 ; 1}(x) * v(x+\varepsilon)-\frac{2}{n !} a_{0, n ; 0}(x) \\
& n a_{1, n ; 1}(x)=a_{1, n-1 ; 0}(x+\varepsilon)+u(x) * a_{1, n-1 ; 1}(x)+v(x) * a_{1, n-1 ; 2}(x-\varepsilon)-\frac{2}{n !} a_{0, n ; 1}(x) \\
& =a_{1, n-1 ; 0}(x)+a_{1, n-1 ; 1}(x) * u(x+\varepsilon)+a_{1, n-1 ; 2}(x) * v(x+2 \varepsilon)-\frac{2}{n !} a_{0, n ; 1}(x) .
\end{aligned}
$$

From the eq.(4.41), we can derive the following equation by which one can represent $a_{1, n-1 ;-1}$ in terms of other functions

$$
(\Lambda-1) a_{1, n-1 ;-1}(x)=a_{1, n-1 ; 1}(x) * v(x+\varepsilon)-v(x) * a_{1, n-1 ; 1}(x-\varepsilon)+\left[a_{1, n-1 ; 0}(x), u(x)\right]_{*} .
$$

We need to notice that there is one more term than the commutative system, i.e. the term $\left[a_{1, n-1 ; 0}(x), u(x)\right]_{*}$. This further leads to the following recursion relation between two Poisson brackets 


$$
\begin{aligned}
& \left\{u, H_{1, n}\right\}_{2} \\
= & \frac{1}{\varepsilon}\left[\Lambda R_{v}-v * \Lambda^{-1}+R_{u} *\left(\frac{\Lambda}{\Lambda-1} R_{u}\right)\right. \\
& -\left(\frac{\Lambda}{\Lambda-1} u * R_{u}-u *(\Lambda-1)^{-1} R_{u}+u *(\Lambda-1)^{-1} u\right] a_{1, n-1 ; 0}(x) \\
& +\frac{1}{\varepsilon}\left[R_{u} \frac{\Lambda}{\Lambda-1} R_{v(x+\varepsilon)}-R_{u}\left[\frac{\Lambda}{\Lambda-1} v * \Lambda^{-1}\right]\right. \\
& \left.-u(\Lambda-1)^{-1} R_{v(x+\varepsilon)}+u(\Lambda-1)^{-1} v \Lambda^{-1}\right] a_{1, n-1 ; 1}(x) \\
= & \frac{1}{\varepsilon}\left\{a_{1, n-1 ; 0}(x+\varepsilon) * v(x+\varepsilon)-v(x) * a_{1, n-1 ; 0}(x-\varepsilon)+u(x) * a_{1, n-1 ; 1}(x) * v(x+\varepsilon)\right. \\
& -v(x) * a_{1, n-1 ; 1}(x-\varepsilon) * u(x)+a_{1, n-1 ;-1}(x+\varepsilon) * u(x)-u(x) * a_{1, n-1 ;-1}(x) \\
& \left.+v(x) * a_{1, n-1 ; 1}(x-\varepsilon) * u(x)-u(x) * a_{1, n-1 ; 1}(x) * v(x+\varepsilon)\right\} \\
= & \frac{n}{\varepsilon}\left\{a_{1, n ; 1}(x) * v(x+\varepsilon)-v(x) * a_{1, n ; 1}(x-\varepsilon)+\left[a_{1, n ; 0}(x), u(x)\right]_{*}\right\} \\
& +\frac{2}{\varepsilon n !}\left\{a_{0, n ; 1}(x) * v(x+\varepsilon)-v(x) * a_{0, n ; 1}(x-\varepsilon)+\left[a_{0, n ; 0}(x), u(x)\right]_{*}\right\} \\
= & n\left\{u, H_{1, n}\right\}_{1}+\frac{2}{n !}\left\{u, H_{0, n}\right\}_{1} .
\end{aligned}
$$

The similar recursion flow on the noncommutative function $v$ can be similarly derived by the following calculation,

$$
\begin{aligned}
&\left\{v, H_{1, n}\right\}_{2} \\
&=\frac{1}{\varepsilon}\left[R_{v} \Lambda^{2}(\Lambda-1)^{-1} R_{v}-R_{v} \frac{\Lambda}{\Lambda-1} v-v(\Lambda-1)^{-1} R_{v}+v \Lambda^{-1}(\Lambda-1)^{-1} v\right] a_{1, n-1 ; 1}(x-\varepsilon) \\
&+\frac{1}{\varepsilon}\left[u(x) R_{v}-v(x) \Lambda^{-1} R_{u}+R_{v} \frac{\Lambda}{\Lambda-1} R_{u}\right. \\
&\left.-R_{v} \frac{\Lambda}{\Lambda-1} u-v \Lambda^{-1}(\Lambda-1)^{-1} R_{u}+v \Lambda^{-1}(\Lambda-1)^{-1} u\right] a_{1, n-1 ; 0}(x) \\
&= \frac{1}{\varepsilon}\left\{u(x) * a_{1, n-1 ; 0}(x) * v(x)-v(x) * a_{1, n-1 ; 0}(x-\varepsilon) * u(x-\varepsilon)\right. \\
&+\left[\frac{\Lambda}{\Lambda-1}\left(a_{1, n-1 ; 1}(x) * v(x+\varepsilon)-v(x) * a_{1, n-1 ; 1}(x-\varepsilon)+\left[a_{1, n-1 ; 0}(x), u(x)\right]_{*}\right)\right] v(x) \\
&\left.-v(x) \Lambda^{-1}(\Lambda-1)^{-1}\left(a_{1, n-1 ; 1}(x) * v(x+\varepsilon)-v(x) * a_{1, n-1 ; 1}(x-\varepsilon)+\left[a_{1, n-1 ; 0}(x), u(x)\right]_{*}\right)\right\} \\
&= \frac{1}{\varepsilon}\left\{u(x) * a_{1, n-1 ; 0}(x) * v(x)-v(x) * a_{1, n-1 ; 0}(x-\varepsilon) * u(x-\varepsilon)\right. \\
&\left.+a_{1, n-1 ;-1}(x+\varepsilon) * v(x)-v(x) * a_{1, n-1 ;-1}(x-\varepsilon)\right\} \\
&= \frac{n}{\varepsilon}\left[a_{1, n ; 0}(x) * v(x)-v(x) * a_{1, n ; 0}(x-\varepsilon)\right]+\frac{2}{\varepsilon n !}\left[a_{0, n ; 0}(x) * v(x)-v(x) * a_{0, n ; 0}(x-\varepsilon)\right] \\
&= n\left\{v, H_{1, n}\right\}_{1}+\left\{v, H_{0, n}\right\}_{1} .
\end{aligned}
$$

The theorem is proved till now. 
Now similarly as [3], sometimes the above mentioned conserved quantities are not real conserved quantities. To find the real conserved density $\sigma$ which satisfies

$$
\partial_{t} \sigma=\partial_{x} J
$$

where $J$ is called the conserved flux, we can do the following calculation

$$
\begin{aligned}
\frac{\partial h_{k, j}}{\partial t_{m, i}} & =\sum_{p}\left[a_{m, i, p} \Lambda^{p}, a_{k, j,-p} \Lambda^{-p}\right]_{*} \\
& =\sum_{p} a_{m, i, p}(x) * a_{k, j,-p}(x+p \varepsilon)-a_{k, j,-p}(x) * a_{m, i, p}(x-p \varepsilon) \\
& =\sum_{p}\left(1-\Lambda^{-p}\right)\left[a_{m, i, p}(x) * a_{k, j,-p}(x+p \varepsilon)\right]-\sum_{p}\left[a_{k, j,-p}(x), a_{m, i, p}(x-p \varepsilon)\right]_{*} .
\end{aligned}
$$

Because

$$
\begin{aligned}
& \sum_{p}\left[a_{k, j,-p}(x), a_{m, i, p}(x-p \varepsilon)\right]_{*} \\
= & -\sum_{p} \theta^{i j} \partial_{t_{i}}\left(a_{k, j,-p}(x) \diamond \partial_{t_{j}} a_{m, i, p}(x-p \varepsilon)\right)-\sum_{p} \Omega^{i j} \partial_{s_{i}}\left(a_{k, j,-p}(x) \diamond \partial_{s_{j}} a_{m, i, p}(x-p \varepsilon)\right) \\
= & -\theta^{i j} \partial_{t_{i}} \operatorname{Res}\left(B_{k, j} \diamond \partial_{t_{j}} B_{m, i}\right)-\Omega^{i j} \partial_{s_{i}} \operatorname{Res}\left(B_{k, j} \diamond \partial_{s_{j}} B_{m, i}\right),
\end{aligned}
$$

therefore if we identify the coordinate $t_{0, i}=t_{i}$ with time $t$, we get the real conserved density as follows

$$
\sigma=h_{k, j}-\theta^{i j} \partial_{t_{i}} \operatorname{Res}\left(B_{k, j} \diamond \partial_{t_{j}} B_{0, i}\right),
$$

where the suffices $j$ must run in the space-time directions only. If we identify the coordinate $t_{1, i}=s_{i}$ with time $t$, we get the real conserved density as follows

$$
\sigma=h_{k, j}-\Omega^{i j} \partial_{s_{i}} \operatorname{Res}\left(B_{k, j} \diamond \partial_{s_{j}} B_{1, i}\right),
$$

where the suffices $j$ must run in the space-time directions only. For the ENTH, we identify the spatial coordinate $s_{0}=x$, the coordinate $t_{1, i}=s_{i}$ with time $t$, and so we can get the real conserved density as follows

$$
\sigma=h_{k, j}-\Omega^{i 0} \partial_{s_{i}} \operatorname{Res}\left(B_{k, 0} \diamond \partial_{s_{0}} B_{1, i}\right) .
$$

We can easily see in the case of space-space noncommutativity, the conserved density is given by the $h_{k, j}$ as the commutative case. The deformation terms will appear in the second term of Eq. (4.46) in the case of space-time noncommutativity.

Remark 4.2. In the case of space-space noncommutativity, the conserved density is given by the residue of powers of the Lax operator and the integration will take only in spatial directions without time directions as the commutative case.

By doing the reductions on Lie algebras, we can derive the following remark.

Remark 4.3. The Lax equation and bi-hamiltonian structure of extended noncommutative Toda hierarchy will be reduced the ones of the extended Toda hierarchy in [11] when $\theta=\Omega=0$, extended multicomponent Toda hierarchy in [24] when $\theta=\Omega=0$ and $\mathfrak{g}=g l_{N}(\mathbb{C})$, extended $Z_{N}$-Toda hierarchy in [25] when $\theta=\Omega=0$ and the algebra takes values in a maximum commutative subalgebra 
of $g l_{N}(\mathbb{C})$, extended $q$-Toda hierarchy in [26] when $\theta=\Omega=0$ and the algebra takes values in a $q$-shift algebra respectively.

\section{Conclusions and Discussions}

In this paper, we constructed a new hierarchy called extended noncommutative Toda hierarchy(ENTH) and extended Sato theory to this hierarchy including Sato equations, matrix wave operators and bi-Hamiltonian. Similarly as the extended Toda hierarchy and extended bigraded Toda hierarchy in Gromov-Witten theory of $\mathbb{C} P^{1}$ and orbiford respectively, this noncommutative hierarchy deserves further studying and exploring because of its potential applications in noncommutative quantum fields and Gromov-Witten theory.

\section{Acknowledgements}

Chuanzhong Li is supported by the Zhejiang Provincial Natural Science Foundation under Grant No. LY15A010004, the National Natural Science Foundation of China under Grant No. 11571192, the Natural Science Foundation of Ningbo under Grant No. 2015A610157 and the K. C. Wong Magna Fund in Ningbo University.

\section{References}

[1] M. Hamanaka, Noncommutative Integrable Systems and Quasideterminants, Arxiv:1012.6043.

[2] M. R. Douglas and N. A. Nekrasov, Noncommutative Field Theory, Rev. Mod. Phys. 73 (2002), 977.

[3] M. Hamanaka, Commuting flows and conservation laws for noncommutative Lax hierarchies, J. Math. Phys. 46(2005), 052701.

[4] A. Dimakis, F. Muller-Hoissen, Explorations of the extended ncKP hierarchy, J. Phys. A 37 (2004) 10899.

[5] W. X. Ma, An extended noncommutative KP hierarchy, Journal of Mathematical Physics, 51(2010), 073505 .

[6] M. Sakakibara, Factorization methods for Noncommutative KP and Toda hierarchy, J. Phys. A 37(2004), L599-L604.

[7] M. Toda, Vibration of a chain with nonlinear interaction. J. Phys. Soc. Jpn. 22(1967), 431-436.

[8] M. Toda, Nonlinear waves and solitons(Kluwer Academic Publishers, Dordrecht, Holland, 1989).

[9] W. X. Ma, K. Maruno, Complexiton solutions of the Toda lattice equation, Physica A, 343(2004), 219237.

[10] K. Ueno, K. Takasaki, Toda lattice hierarchy, In "Group representations and systems of differential equations" (Tokyo, 1982), 1-95, Adv. Stud. Pure Math., 4, North-Holland, Amsterdam, 1984.

[11] G. Carlet, B. Dubrovin, Y. Zhang, The Extended Toda Hierarchy, Moscow Mathematical Journal 4 (2004), 313-332.

[12] T. Milanov, Hirota quadratic equations for the extended Toda hierarchy, Duke Math. J. 138 (2007), 161-178.

[13] B. Dubrovin, Y. Zhang, Virasoro symmetries of the extended Toda hierarchy Commun. Math. Phys. 250(2004), 161-93.

[14] T. Eguchi, S. K. Yang, The topological $\mathbb{C} P^{1}$ model and the large-N matrix integral, Mod. Phys. Lett. A 9(1994), 2893-902.

[15] G. Carlet, The extended bigraded Toda hierarchy, J. Phys. A, 39 (2006), 9411-9435.

[16] C. Z. Li, J. S. He, K. Wu, Y. Cheng, Tau function and Hirota bilinear equations for the extended bigraded Toda Hierarchy, J. Math. Phys.51(2010),043514.

[17] C. Z. Li, Solutions of bigraded Toda hierarchy, J. Phys. A, 44(2011), 255201.

[18] C. Z. Li, J. S. He, Dispersionless bigraded Toda hierarchy and its additional symmetry, Reviews in Mathematical Physics, 24(2012), 1230003. 
[19] C. Z. Li, J. S. He, Y. C. Su, Block type symmetry of bigraded Toda hierarchy, J. Math. Phys. 53(2012), 013517.

[20] J. E. Moyal, Quantum mechanics as a statistical theory, Proc. Cambridge Phil. Soc. 45 (1949), 99.

[21] A. Dimakis, F. Muller-Hoissen, Extension of Moyal-deformed hierarchies of soliton equations, arXiv: nlin/0408023.

[22] P. Etingof, I. Gelfand and V. Retakh Factorization of differential operators, quasideterminants, and nonabelian Toda field equations, Mathematical Research Letters 4(1997), 413-425.

[23] K. M. Lee, Chern-Simons Solitons, Chiral Model, and (affine) Toda Model on Noncommutative Space, J. High Energy Phys. 08(2004), 054.

[24] C. Z. Li, J. S. He, On the extended multi-component Toda hierarchy, Math. Phys. Anal. Geom. 17(2014), 377-407.

[25] C. Z. Li, J. S. He, The extended $Z_{N}$-Toda hierarchy, arXiv:1403.0684, Theor. Math. Phys. 185(2015), 1614-1635

[26] C. Z. Li, Sato theory on the $q$-Toda hierarchy and its extension, Chaos, Solitons and Fractals 76 (2015), $10-23$.

[27] M. Blaszak, B. M. Szablikowski, Classical R-matrix theory of dispersionless systems: I. $(1+1)$ dimension theory, J. Phys. A, 35 (2002) 10325-10344.

[28] W. X. Ma, B. Fuchssteiner, Integrable Theory of the Perturbation Equations, Chaos, Solitons Fractals, 7(1996), 1227-1250. 